\title{
Highly Multistable Composite Surfaces
}

\author{
Yuefeng Cui ${ }^{\mathrm{a}}$, Matthew Santer ${ }^{\mathrm{a}, *}$ \\ ${ }^{a}$ Department of Aeronautics, Imperial College London, London, SW7 2AZ, UK
}

\begin{abstract}
Novel continuous composite surfaces are presented which possess a high degree of multistability. Inspired by the illustrative behaviour of a multistable analog model, we first show how two identical bistable composite shells with tailored asymmetric bistability may be connected to form a continuous quadstable surface. The concept is then extended to surfaces composed of three and by extension more identical bistable shells connected in series in order to achieve additional stable states. The multistable behaviour of these surfaces is investigated by finite element analysis and verified by experimental work.
\end{abstract}

Keywords:

Multistable surfaces, Composites, Adaptive structures

\section{Introduction}

Due to their multiple discrete stable configurations, compliant multistable surfaces have been considered for use in many adaptive applications. They offer several advantages when used as structures requiring shape variation, including the reduction in required components and an increase in their potential operational environments [1]. The increasingly high demand for adaptive structures across many fields of engineering, but in particular aerospace, including flow control and adaptive optics, makes research into extending the the degree of multistability, and hence the adaptivity, timely.

It has long been known composite laminates with unsymmetric layups may present multiple stable configurations at room temperature [2, 3]. Due to

\footnotetext{
*Corresponding author.

Address: South Kensington Campus, London SW7 2AZ, UK

Tel.: +44(0)20 75945117

Email address: m.santer@imperial.ac.uk (Matthew Santer)
} 
the mismatch of coefficients of thermal expansion in the directions axial and transverse to the fiber, residual thermal stresses build up during the curing process. These residual stresses cause the plates to curve into one of two possible stable cylindrical shapes after curing. In addition, each cylindrical shape can transition to the other by means of an applied external actuation.

However, an individual bistable composite laminate normally cannot fulfill the requirements of real world applications. On the one hand, bistable composite shells are required to be connected with other components, on the other hand, adaptive applications may need more than two stable configurations. Therefore, the extension of previous studies of bistable plates to achieve multistable structures composed of multiple bistable composite shells is a subject of interest. For example, Mattioni et al. [4, 5] connected one edge of a bistable composite shell to a symmetric, i.e. monostable, laminate to demonstrate the use of bistable composites is feasible in morphing structures. Although the movement of one edge of the bistable laminate is restrained, the compound surface demonstrates two discrete stable configurations. However, when two edges of a bistable composite laminate are clamped by monostable laminates, the plate only demonstrate one stable configuration. To regain multistability, a designed surface consisting of symmetric and unsymmetric laminate sections may be introduced, for example, the shell demonstrated by Arrieta et al. [6]. The embedded composite shell with variable stiffness can demonstrate bistability and avoid the conventional connections which may increase the risk of laminate failure. However, these embedding designs cannot increase the number of stable configurations. To achieve high degrees of multistability, Dai et al. [7] fabricated tristable composite lattices by connecting four bistable rectangular laminates with discrete joints which were then assembled $n$ lattice cells by bolts. The lattice structure can present $2^{n}$ stable shapes. A similar design is demonstrated in [8]. These attempts to achieve highly multistable structures have two main disadvantages: first, large numbers of components are used - this negates one of the principle advantages of the use of compliant mechanism in adaptive system; second, bolted connections may reduce the performance and lifetime of composites.

Until now, to the authors' knowledge there has been no demonstration of a highly multistable continuous composite surface fabricated without any conventional fixation. In this paper, novel multistable composite surfaces are constructed by connecting several identical bistable composite shells in series. As previously stated, if $n$ bistable components are connected, the resulting system may exhibit up to $2^{n}$ discrete stable configurations [9]. This 
has previously been demonstrated for systems where the individual bistable components are decoupled and may be independently actuated via the design of statically and kinetically-determinate systems [1]. When components are connected in a continuous sense, however, it is essential to consider their interaction by means of the coupling along common boundaries. In this paper we begin this investigation by considering the interaction between two bistable plates and then extend this to the study of three connected bistable plates.

Furthermore, in order to interpret the multistable behaviour of these newly designed surfaces, a two-dimensional multistable analog model will be introduced first and a parametric study will be carried out to characterise the controlling parameters which determine multistability of the analog model. Inspired by the understanding gained from this simplified study, a design method is developed to assist the compound surface achieving higher multistability. Since each of these shells possesses two stable states, the compound structure can theoretically present up to a maximum of eight stable configurations. However, due to the interaction between the connected shells, it will be shown that a maximum of seven discrete stable states can be achieved in a surface consisting of three connected square bistable composite shells.

\section{Tripled Bistable Structures}

Before analysing the behaviour of connected bistable composite shells, it is instructive to consider an analog model consisting of three biased von Mises truss systems to understand the general behaviour of bistable elements connected in series. The three von Mises trusses are connected in series by rigid bars and coupling springs. By varying key parameters, the multistable behaviour of the truss structure is controlled and a maximum eight discrete stable states, as expected, are found to exist.

As illustrated in Fig. 1, the three identical von Mises trusses consist of elastic rods of initial length $L_{0}$. The span width of each truss system is $2 l$. The axial stiffness of the rods is $K_{s}$. Biasing springs $K_{b}$ are attached to the centrally-located hinges A, B and C. Any two adjacent truss systems are connected as shown by a rigid bar and coupling spring $K_{c}$. All of the trusses are shown in their initial strain free stable configurations. The initial height of the truss members is defined by the vertical distance $h$ and the maximum displacement of a truss is $2 h$. 
We first investigate the bistability of a single truss. When a vertical displacement $\delta$ is applied, the rods will be compressed and the truss system will move downward. Meanwhile the strain energy stored by the truss will rise. When the displacement reaches a critical value, the strain energy will reach a maximum value and will drop as the displacement is increased. As the truss is displacement controlled there is no dynamic jump to the second stable state. This truss system then continues to a new stable configuration corresponding to a second energy minimum (see Fig. 2). The relationship between the displacement relative to double height $2 h$ and the stored strain energy relative to the largest local strain energy minimum of a bistable truss achieved is presented in the Appendix. Fig. 3 demonstrates the relationship of the relative displacement and relative strain energy of a von Mises truss with increasing $K_{b}$, in which $100 \%$ relative displacement indicates the vertical displacement of the truss is the maximum displacement, $2 h$. The two local strain energy minima correspond to two stable states. When $K_{b}=0$, the two local minimum values are identical, and the system is symmetrically bistable. With $K_{b}$ increasing, the actuation energy of one stable state rises and the energy gap between two stable states increases. The stable state possessing lower potential energy becomes the preferred stable state. When $K_{b}$ reaches a critical value, the second energy minimum disappears and the system becomes monostable. In other words, to ensure that each individual truss is bistable, the stiffness of the bias spring $K_{b}$ must be lower than a critical value.

Besides the stiffness of biasing spring $K_{b}$, the bistability of the von Mises truss is also determined by $K_{s}$. Fig. 4 illustrates the influence of $K_{s}$ on the stored strain energy of the bistable truss. With $K_{s}$ increasing, higher energy is required for actuation. This means stiffer rods cause the stable configurations of the truss to be more stable in the sense that greater energy input is required to transition between the states.

After understanding the bistability of an individual von Mises truss, the next step is to study the influences of these key parameters on the multistable behaviour of connected truss systems. When one truss of this connected structure is actuated by displacement control, the motion will be transmitted to other driven trusses by the rigid bars and connecting springs. If the vertical displacement of point $A, B, C$ is denoted by $\delta_{1}, \delta_{2}, \delta_{3}$, the loads applied by 
the connecting spring on point $A, B, C$ are expressed as

$$
\begin{aligned}
& P_{1}=K_{c}\left(\delta_{2}-\delta_{1}\right) ; \\
& P_{2}=K_{c}\left(\delta_{1}-\delta_{2}\right)+K_{c}\left(\delta_{3}-\delta_{2}\right) ; \\
& P_{3}=K_{c}\left(\delta_{2}-\delta_{3}\right) .
\end{aligned}
$$

The equilibrium path $P_{i}\left(\delta_{i}\right)$ of these three systems must also be equal to

$$
P_{i}=K_{b} \delta_{i}-2 K_{s} X_{i} \sin \theta_{i}, i=1,2,3
$$

in which $X_{i}$ are the compressive deformations of the diagonal members and $\theta_{i}$ are the angles between a hinge and the horizontal direction. They can be obtained by

$$
\begin{array}{r}
X_{i}=L_{0}-\sqrt{l^{2}+\left(h-\delta_{i}\right)^{2}}, i=1,2,3 \\
\theta_{i}=\arctan \left(\frac{h-\delta_{i}}{l}\right), i=1,2,3
\end{array}
$$

Combining Eqs. 7, 2 and 3, it may be seen that the force-controlled displacement of the two driven trusses may be expressed as a function of the imposed displacement $\delta$ on the driving truss.

In order to identify each state clearly, we use a binary notation. The original stable state of the truss is denoted as 0 and the actuated stable state is denoted as 1 . Stable state 000 and 111 are two primary stable states since there is no interaction between the connected trusses. When not all connected trusses are in the same state, the tripled truss structure is in an intermediate configuration. In an intermediate state, the interaction between two adjacent trusses within different states may be large enough to trigger one of the trusses to its other state; in other words, this intermediate state is not stable. To determine the stability of intermediate states, four actuation steps are applied. Specifically:

- step A: actuate from state 000 to state 100;

- step B: actuate from state 100 to state 110;

- step C: actuate from state 110 to state 010; 
- step D: actuate from state 100 to state 101.

States 001 and 011 are not investigated as their behaviour may be inferred due to the symmetry of the system. In all cases the actuated truss is subjected to displacement control, and the other two trusses are in a force-controlled regime.

The tripled von Mises truss structure possesses eight stable states if and only if the actuation of the driving truss does not actuate the other two trusses in every actuation step. Fig. 5 demonstrates the relative displacements of the trusses of an octo-stable truss structure by the four actuation steps. It is clear that, in each actuation step, the relative displacements of the driven trusses are small then the structure reaches a new stable state. It indicates that no automatic snapthrough occurs and the expected stable state can be achieved. The energy graphs of the tripled truss structure (see Fig. 6) verify the stability of these new states. It is clear that the structure reaches a local energy minimum at the new state.

The analytical results show the multistability of the tripled von Mises trusses is determined by the connection between the trusses. If the value of $K_{c}$ rises, the interaction between connected trusses will also increase. If $K_{c}$ exceeds a critical value, the tripled truss systems will not demonstrate eight stable states. Fig. 7 illustrates the relative displacements of the trusses of a hepta-stable truss structure by the four actuation steps. In the actuation step C, the movement of truss 1 results in increasing external strain energy introduced to truss 2. Before truss 1 is fully actuated, the introduced energy to truss 2 already exceeds the actuation energy and the truss snaps back to state 0 . In other words, the intermediate state 010 of this structure is no longer a stable state. By actuating truss 1, the structure will jump through from state 110 to state 000 directly. Thus, this structure only possesses seven stable states. As $K_{c}$ increases, more intermediate states become switched off via a similar mechanism.

To avoid the unwanted automatic actuation, the actuation energy of the truss needs to be raised. According to the investigation of the multistability of the single truss, the actuation energy is determined by the relative stiffness of the bar, $K_{s}$. By increasing the value of $K_{s}$, the stable state 010 is achievable again and the tripled truss structure regains octo-stability successfully. In other words, higher $K_{s}$ can help the tripled trusses with strong connection to achieve higher multistability.

Although the bias stiffness $K_{b}$ can affect the degree of asymmetric bista- 
bility of a single truss system, the influence of the value of $K_{b}$ on achieving the octo-stability of this tripled structure is limited. This is because, despite resulting in a more stable state 0 , the increasing $K_{b}$ will lead the actuated state 1 to be less stable. Therefore, in order to ensure the tripled von Mises trusses possesses more stable states, these bistable trusses should not be highly asymmetrically bistable.

In summary, the response of the tripled bistable trusses shows that its multistability is determined by the relative stiffness of the bias and coupling springs and the bars. Specifically:

- as the value of bias stiffness $K_{b}$ increases from 0 , the single truss changes from being symmetrically bistable to being asymmetrically bistable. If $K_{b}$ is over a critical value, the truss will be monostable;

- higher coupling stiffness $K_{c}$ will lead to higher actuation energy of the individual truss;

- to avoid the actuation of the driven truss caused by the driving truss, in other words, to ensure the system achieves octostability, the relative stiffness of the connecting spring $K_{c}$ must be lower than a critical value;

- the critical value of $K_{c}$ is dependent on the value of $K_{s}$ i.e. the symmetry of bistability of each individual truss. The critical value of $K_{c}$ can be raised by increasing the relative stiffness of the rods, $K_{s}$;

- a structure composed of three symmetrically bistable trusses is more likely to achieve octostability than those composed of asymmetrically bistable trusses.

\section{Connected Bistable Composite Shells}

The understanding gained from the previous section can assist in interpreting the response of the novel designed surfaces. We restrict the number of bistable units of the multistable structure to be up to three in this paper, however, this concept can be easily extended to a multistable surface consisting of many more bistable shells connected by the same approach.

The composite shells investigated in this paper are made of Hexcel IM7/8552 Graphite/Epoxy Prepreg. The mechanical properties are taken from the manufacture's data sheet and listed in Table 1. Whilst restricting the design 
space, this enables the behaviour to be validated by experimental models. The stacking sequence is $[0 / 90]$ to ensure that the laminates have a moderate out-of-plane displacement which would help the connected shells to demonstrate more stable states and avoiding the generation of twisting curvature after curing. For the sake of easy identification, we continue to use binary notation to represent the states. If a shell curves along the linking edge direction, the shell is defined as being in state 0 ; otherwise, it is in state 1.

It is noted that a Ritz energy analysis is often considered a fast and reliable approach when investigating the multistable behaviour of unsymmetric composite laminates $[10,11,12]$. However, this approach is not adopted in this paper due to the large number of terms in the approximating polynomials required to adequately represent the coupled shells. The significantly increased calculation time and the presence of many local minima make a Ritz approach no longer a feasible way of investigating connected bistable composite shells. Therefore, Finite Element Analysis (FEA) is exclusively used as the simulation method in this research.

\subsection{Coupled bistable composite shells}

Before considering the behaviour of the tripled connected bistable shells, we first investigate the multistable behaviour of coupled bistable shells. This is because, although the multistable behaviour of the tripled bistable truss structure has been understood, the nature of the interaction between fully connected shells is significantly more complex than the one dimension connection which is used in the analog model. For a coupled bistable shell structure, a maximum of four stable states may be present. Among these four stable states, the two primary states exist in which both of the shells have the same stable configuration. Besides two primary stable states, the connected plates may achieve stability during intermediate states in which the two shells individually present different configurations. Due to the continuous connection, the two plates will be subject to considerable deformations which may trigger the surface jump to the primary stable state during an attempted transition to an intermediate state. Thus, the key to achieving highly multistable surfaces is the stability of intermediate states.

FEA is performed with the commercial software SAMCEF V13.1. Following a mesh refinement study, a single plate is simulated by 400 8-node square shell elements. This level of discretisation has been shown to provide mesh 
independent solutions. Inertial phenomena are neglected in this study; a geometrically nonlinear static analysis strategy is therefore adopted. The two shells are connected continuously along a common boundary. The manufacturing process is simulated first by imposing a ramped temperature increase of $160^{\circ} \mathrm{C}$. After manufacturing, the actuation process is implemented by applying a controlled displacement to the driving shell until the shell snaps through to another stable state. Meanwhile, the central point and two free vertices of the driven shell have to be fixed for avoiding rotation (see Fig. 8.a). The deformation will transmit to the driven shell through the common edge. After the actuation, the fixed vertices are released and if the driven shell does not snap to a new stable configuration and the driving shell maintains its new shape (see Fig. 8.b), the intermediate state is deemed stable and the connected shells determined to have four stable states. Otherwise, the intermediate state is not stable and the structure will jump to a new primary stable state (see Fig. 8.c). In this case the compound surface remains bistable.

According to the analog model, the degree of bistable asymmetry influences the multistable behaviour of the connected trusses. Considering the corresponding effect for coupled composite shells, biasing strips made by symmetric $[0 / 0]$ laminates are attached on the center of bistable composite shells to vary the asymmetric bistability of the composite shell. To investigate the influence of attaching biasing strips on the bistability of composite shells, the strips may be attached parallel or perpendicularly to the linking edges respectively (see Fig. 9). The influence of biasing strips on the bistable behaviour of a single shell is shown in Fig. 10. As expected, by attaching the biasing strip, the two stable states no longer exist at the same potential energy level. The degree of asymmetric bistability of the single shell increases and the stable state possessing lower potential energy becomes the preferred stable state. It is also noted that the selection of one of the two possible orientations of biasing strip enables either of the stable states to be made preferential.

We consider three cases when the unbiased shell and the two differently biased shells are connected to an identical partner. To demonstrate the stability of intermediate states, the surfaces begin from the primary stable state 00 and are subjected to controlled displacements as the actuation is applied to the two free vertices of one shell. During the actuation procedure, deformations will transmit to the driven shell through the linking edges. With the controlled displacement increasing, the driving shell will snap through to 
the new stable state at a critical point, and the strain energy of the driven shell will rise during this actuation procedure. If a compound surface has stable intermediate states, the driven shell will not snap through with the driving shell and the strain energy of the whole structure will reach a local minimum. It should be noted, due to symmetry, that only one intermediate state is investigated in this study.

Fig. 11 presents the potential energy graph of three different coupled bistable shells during the actuation from the primary stable state 00 to the primary stable state 11 . Since some nodes on the surface need to be temporarily fixed during the actuation to avoid unwanted rotations and buckling, the potential energy curves show some asymmetries, but the local minima in each case are clearly visible. The coupled symmetrically bistable shells and the coupled bistable shells with perpendicular biasing strips only demonstrate two potential energy minima which represent two primary stable states, whereas the coupled bistable shells with parallel biasing strips demonstrate three local energy minima. Besides the two primary stable states, this surface can achieve stable intermediate state and possesses quadstability. By tailoring the asymmetric bistability, coupled bistable composite shells can successfully achieve a higher degree of multistability.

This behaviour may be explained by consideration of the strain energy of the individual bistable units in the coupled system. Fig. 12 corresponds to the case when the two connected bistable plates are symmetrically bistable. The right hand shell has been transitioned via displacement control into state 1 and the left hand shell has deformed to accommodate this transition. It can be seen that the deformation imparted to the driven shell introduces sufficient strain energy to cause it to exit the energy well corresponding to state 0 and upon release will dynamically jump to state 1 . Therefore the only stable equilibrium configuration are states 00 and 11 and it can be seen that there are no stable intermediate configurations. This coupled system is therefore bistable.

We now consider the effect of causing state 1 to become energetically preferential by means of the addition of parallel biassing strips. As the degree of bistable asymmetry increases a critical point is reached at which the displacement controlled transition of one shell from state 0 to state 1 causes the other shell to reach the limit point corresponding to the peak of the energy hill. As the bistable asymmetry is further increased, as shown in Fig. 13, the driven shell no longer exits the energy well of state 0 and consequently when displacement control is released the coupled system may adopt a stable in- 
termediate state in which both shells adopt a configuration corresponding to the average strain energy of the two displacement-controlled configurations. This coupled system is therefore quadstable.

\subsection{Tripled bistable composite shells}

Having understood the multistable behaviour of two connected bistable shells, we now generalise to a multistable surface composed of a series of coupled bistable composite shells. We focus on three connected square bistable shells (see Fig. 14) but the evaluated behaviour is readily extended to surfaces consisting of many more shells connected in series.

Theoretically, a surface composed of three bistable shells may present a maximum of eight stable states. Based on the study of the coupled bistable shells in the previous section, all these shells are made asymmetrically bistable via a biasing strip parallel attached to the linking edges in order to obtain more stable states.

In addition to the two primary stable states 000 and 111, this surface has up to a six intermediate stable states. Four actuation steps are applied to verify the stability of these intermediate states, specifically:

- step A: actuate from state 000 to state 100 ;

- step B: actuate from state 100 to state 110 ;

- step C: actuate from state 110 to state 010;

- step D: actuate from state 100 to state 101.

In each actuation step, a controlled displacement is only applied to the driving shell as the actuation load and the other two driven shells are unconstrained. If and only if no driven shell is actuated automatically, the intermediate state obtained is deemed to be stable. Fig. 15 illustrates the configuration transitions in these steps. Due to symmetry, the shapes of state 001 and state 011 are not illustrated.

The existence of intermediate stable states in two connected asymmetric bistable shells with parallel biasing strips has clearly been demonstrated. The existence of stable intermediate state 100 and 110 of tripled bistable shells may be directly inferred as it involves connecting an identical shell to one of the free edges of coupled bistable shells. Since the newly connected shell is in the same stable state as its adjacent shell, no additional strain energy 
is introduced. The FE results prove this assumption: the two intermediate states are stable.

The two remaining possible states that must be considered are states 010 and 101. In both these cases the central shell is connected to two other shells which are in a different state. Fig. 16 shows the case when the compound surface is in state 010. In this case the energy imparted to the two end shells is insufficient to cause them to exit the energy well corresponding to state 0 . Consequently when displacement control is removed the system will adopt the stable equilibrium configuration 010 .

The case where the surface is in state 101 is shown in Fig. 17. As a result sufficient energy is imparted to the central shell to exit the energy well corresponding to state 0 . When the displacement control is removed the system will dynamically jump to state 111 and consequently state 101 does not correspond to a stable equilibrium configuration.

We now consider the reason behind the instability of state 101 in greater detail. It can be observed, with reference to Figs. 12 and 13 that when a shell in state 0 is connected to a shell in state 1 , on release from displacement control a much greater proportion of the strain energy is transferred to the shell with the initially-curved common edge (state 0). This means that in state 101 a large proportion of the strain energy is transferred to the middle shell which is always sufficient to trigger snapthrough. The resistance of the middle shell to snapthrough may be increased through the imposition of asymmetric bistability, however, the second stable state is always annihilated before the dynamic snapthrough is overcome.

\section{Experimental Investigation}

An experimental investigation is carried out to verify the above conclusions. Both composite shells with and without biasing strips are fabricated. To ensure the shells have continuous, robust and smooth connections, the compound shells are fabricated directly as a whole rectangular shell. Because this research investigates only the number of stable configurations of composite shells, the comparison between the numerical results and the experimental results focuses on the qualitative bistability behaviour. Differences in geometry are the result of manufacturing imperfections and thickness variations.

Fig. 18 illustrates the experimental results of the stable configurations

of compound composite surface. The physical composite surface shows the same number of stable states as expected. The tripled composite shells with 
parallel strips shows seven stable configurations in total. A highly multistable surface fabricated without conventional fixation and presenting smooth curvature changes has been successfully demonstrated.

\section{Conclusions}

Composite surfaces possessing highly stability show high potential for use in adaptive applications. In this paper, inspired by an analog model composed by three bistable von Mises truss systems, surfaces consisting of series connected bistable composite shells are presented. Since the asymmetric bistability of bistable elements is proved to determine the multistability of the whole analog model, the asymmetric bistability of individual shell is tailored by attaching a biasing strip. For coupled bistable composite shells, a quadstable surface is achieved as expected. The multistable surface design is also extended to tripled bistable composite shells which is the basic case of series connected bistable composite shells. By attaching biasing strips parallel to the linking edges, the composite surface demonstrates a higher degree of multistability, specifically seven discrete stable configurations. The investigation into the multistable behaviour of tripled bistable composite shells can be developed to design longer composite surfaces composed of more bistable units.

The primary conclusions of this paper are:

- the tripled biased von Mises truss systems can demonstrate a maximum of eight stable states by varying the degree of asymmetric bistability of the individual units;

- coupled bistable composite shells are demonstrated to possess bistable or quadstable behavior by tailoring the bistable asymmetry of the individual shell;

- three series-connected bistable composite shells with parallel biasing strips may achieve a composite surface possessing seven discrete stable configurations. The theoretically-possible eighth stable state is shown not to exist for square shells.

Continuing work will build on the understanding gained in this research to design and construct surfaces where the individual bistable units are connected to form fully three-dimensional adaptive multistable surfaces. 


\section{Acknowledgements}

The technical staff of the Department of Aeronautics in Imperial College London are gratefully thanked for their assistance on the experimental aspects of this work.

\section{Appendix}

When a vertical displacement $\delta$ is applied to a single biased von Mises truss of the type shown in Fig. 1, the corresponding vertical load $P$ applied at the apex in conditions of static equilibrium is given by

$$
P=K_{b} \delta-2 K_{s}\left(L_{0}-\sqrt{l^{2}+(h-\delta)^{2}}\right) \sin \theta
$$

in which $\theta$ is the angle between a hinge and the horizontal direction; $K_{b}$ is the biasing spring stiffness; $K_{s}$ is the axial stiffness of the rod; $L_{0}$ is the initial length of the rod; $l$ is the half of span width of the truss; and $h$ is the initial height of the truss. The rotation angle $\theta$ is evaluated according to

$$
\theta=\arctan \left(\frac{h-\delta}{l}\right)
$$

The stored strain energy $U$ in the von Mises truss resulting from the imparted displacement is given by

$$
U=\frac{1}{2} P \delta=\frac{1}{2}\left(K_{b} \delta-2 K_{s}\left(L_{0}-\sqrt{l^{2}+(h-\delta)^{2}}\right) \sin \theta\right) \delta
$$

The strain energy is a uniquely determined function of the vertical displacement $\delta$ as the system is energetically conservative. The values of $K_{b}$ and $K_{s}$ determine the relationship between the strain energy $U$ and the vertical displacement $\delta$.

When three identical von Mises trusses are connected by coupling springs $K_{c}$, the loads applied to each truss system from the connecting spring are given by

$$
\begin{aligned}
& P_{1}=K_{c}\left(\delta_{2}-\delta_{1}\right) ; \\
& P_{2}=K_{c}\left(\delta_{1}-\delta_{2}\right)+K_{c}\left(\delta_{3}-\delta_{2}\right) ; \\
& P_{3}=K_{c}\left(\delta_{2}-\delta_{3}\right) .
\end{aligned}
$$


Application of Eq. 4 shows that the equilibrium paths followed by each truss are also equal to

$$
P_{i}=K_{b} \delta_{i}-2\left(L_{0}-\sqrt{l^{2}+\left(h-\delta_{i}\right)^{2}}\right) \sin \theta_{i}, i=1,2,3
$$

where the angles between a hinge and the horizontal direction $\theta$ are

$$
\theta_{i}=\arctan \left(\frac{h-\delta_{i}}{l}\right), i=1,2,3
$$

Combining these equations, it may be seen that the force-controlled displacement of the two driven trusses may be expressed as a function of the imposed displacement $\delta$ on the driving truss. Thus the stored strain energy of the complete system is given by

$$
U=\sum_{i=1}^{3} \frac{1}{2} P_{i} \delta_{i}
$$

[1] Santer M., Pellegrino S., Compliant multistable structural elements, International Journal of Solids and Structures, 45 (24)(2008), pp.6190204

[2] Hyer M. W., Some observations on the cured shape of thin unsymmetric laminates, Journal of Composite Materials, 15 (1981), pp.175-94.

[3] Hyer M. W., The room-temperature shapes of four-layer unsymmetric cross-ply laminates, Journal of Composite Materials, 16 (4)(1982), pp.318-40.

[4] Mattioni F., Weaver P. M., Potter K. D., Friswell M. I., Analysis of thermally induced multistable composite, International Journal of Solids and Structures 45 (2)(2008), pp.657-75.

[5] Mattioni F., Weaver P. M., Friswell M. I., Multistable composite plates with piecewise variation of lay-up in the planform, International Journal of Solids and Structures, 46 (1)(2009), pp.151-64.

[6] Arrieta A., Kuder I., Waeber T., Ermanni P., Varible stiffness characteristics of embeddable multi-stable composites, Composite Science and Technology, 97 (2014), pp.12-18. 
[7] Dai F., Li H., Du S., A multi-stable lattice structure and its snapthrough behavior among multiple states, Composite Structures, 99 (2013), pp.56-63.

[8] Dai F., Li H., Du S., A multi-stable wavy skin based on bi-stable laminates, Composites Part A: Applied Science and Manufacturing, 45 (2013), pp.102-8.

[9] Chirikjian G. S., A binary paradigm for robotic manipulators, Proceedings of the 1994 IEEE International Conference on Robotics and Automation. May 8-13, 1994. Vol. 4. Dept. of Mech. Eng., Johns Hopkins Univ., Baltimore, MD, USA, IEEE Comput. Soc. Press, Los Alamitos, CA, USA, pp. 3063-9.

[10] Dano M. L., Hyer M. W., Room-temperature shape of general unsymmetric laminates, Proceedings of the 1996 11th Technical Conference of the American Society for Composites, October 7-9,1996. Virginia Polytechnic Inst and State, Univ, Blacksburg, United States, Technomic Publ Co Inc, Atlanta, GA, USA, pp. 100-10.

[11] Dano M. L., Hyer M. W., Snap-through of unsymmetric fiberreinforced composite laminates, International Journal of Solids and Structures, 39 (1)(2002), pp.175-98.

[12] Pirrera A., Avitabile D., Weaver P. M., Bistable plates for morphing structures: a refined analytical approach with high-order polynomials, International Journal of Solids and Structures, 47 (25-26)(2010), pp.3412-25.

Table 1: Properties of IM7/8552 carbon fibre composites

\begin{tabular}{cccccccc}
\hline $\begin{array}{c}E_{11} \\
(\mathrm{GPa})\end{array}$ & $\begin{array}{c}E_{22} \\
(\mathrm{GPa})\end{array}$ & $\begin{array}{c}G_{12} \\
(\mathrm{GPa})\end{array}$ & $\nu_{12}$ & $\begin{array}{c}\alpha_{1} \\
\left(10^{-6} /{ }^{\circ} \mathrm{C}\right)\end{array}$ & $\begin{array}{c}\alpha_{2} \\
\left(10^{-6} /{ }^{\circ} \mathrm{C}\right)\end{array}$ & $\begin{array}{c}\text { thickness } \\
(\mathrm{mm})\end{array}$ & $\begin{array}{c}\text { side length } \\
(\mathrm{mm})\end{array}$ \\
\hline 164 & 12 & 5.3 & 0.33 & -0.02 & 31.2 & 0.131 & 100 \\
\hline
\end{tabular}




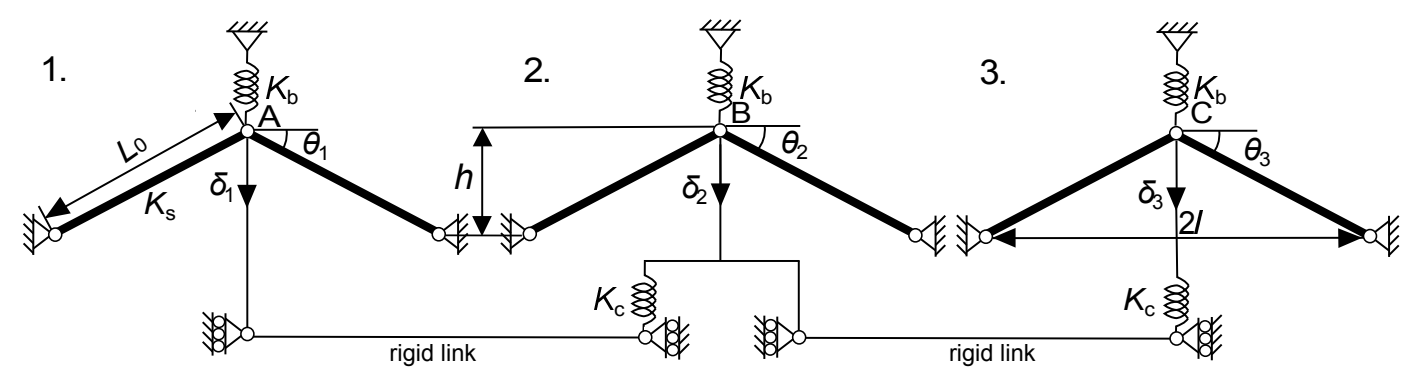

Figure 1: Three connected biased von Mises truss structures



Figure 2: Two stable configurations of a biased von Mises truss 


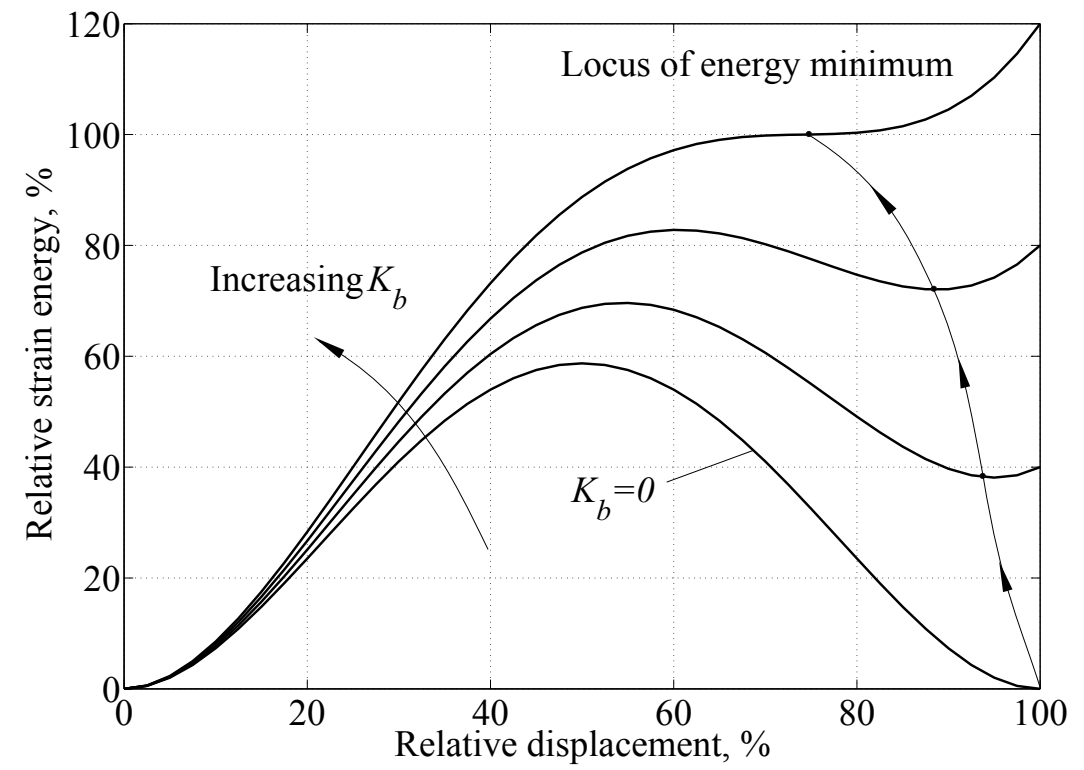

Figure 3: Relative strain energy vs. relative displacement plots for a single von Mises truss with variable bias stiffness $K_{b}$ showing the transition from symmetric to asymmetric bistability 


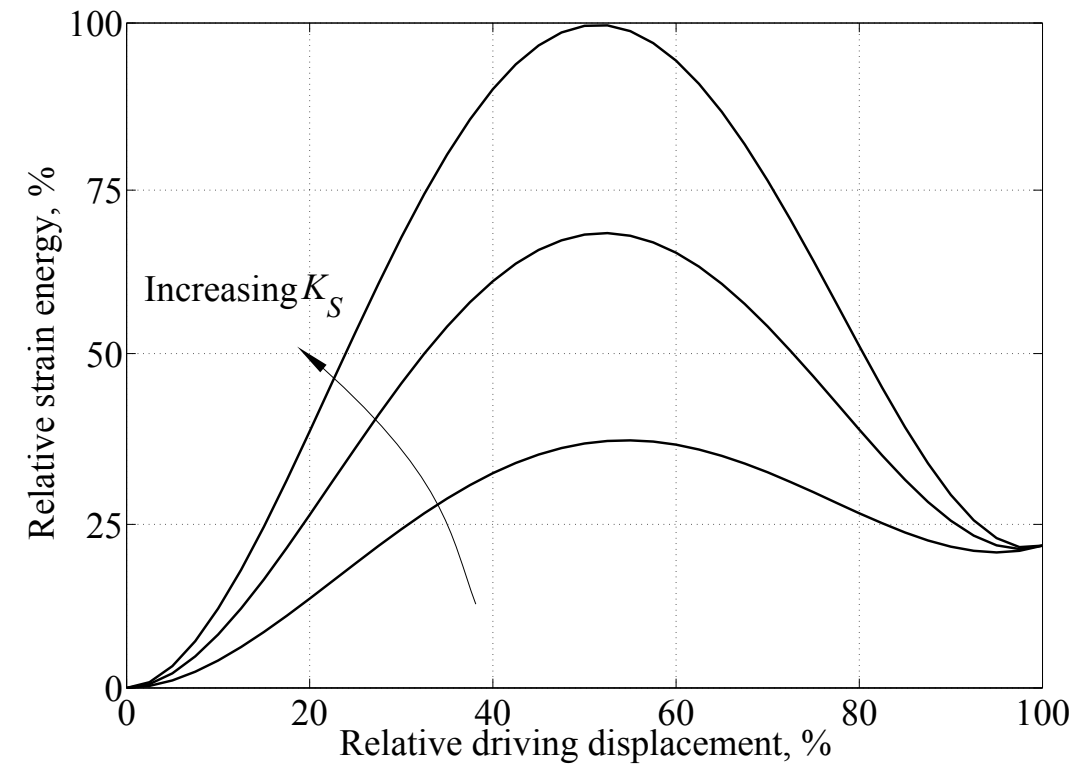

Figure 4: Relative strain energy vs. relative displacement plots for a single asymmetrically bistable $\left(K_{b} \neq 0\right)$ von Mises truss with variable $K_{s}$ 


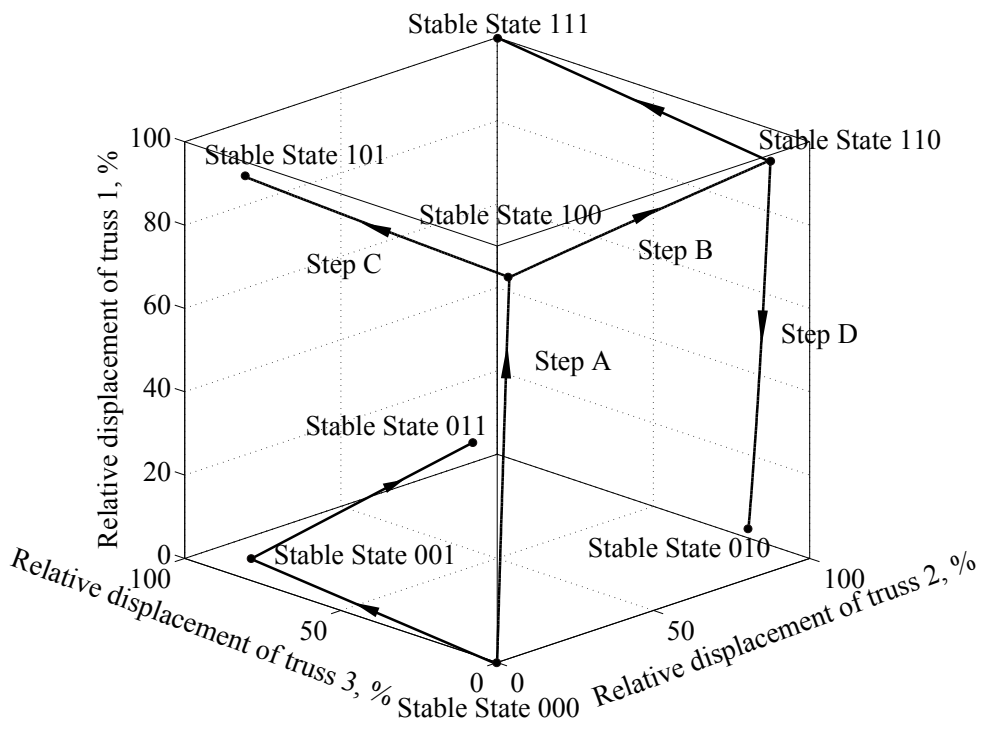

Figure 5: Relative displacement of trusses vs. relative strain energy in different actuation steps plots for the octo-stable truss structure 


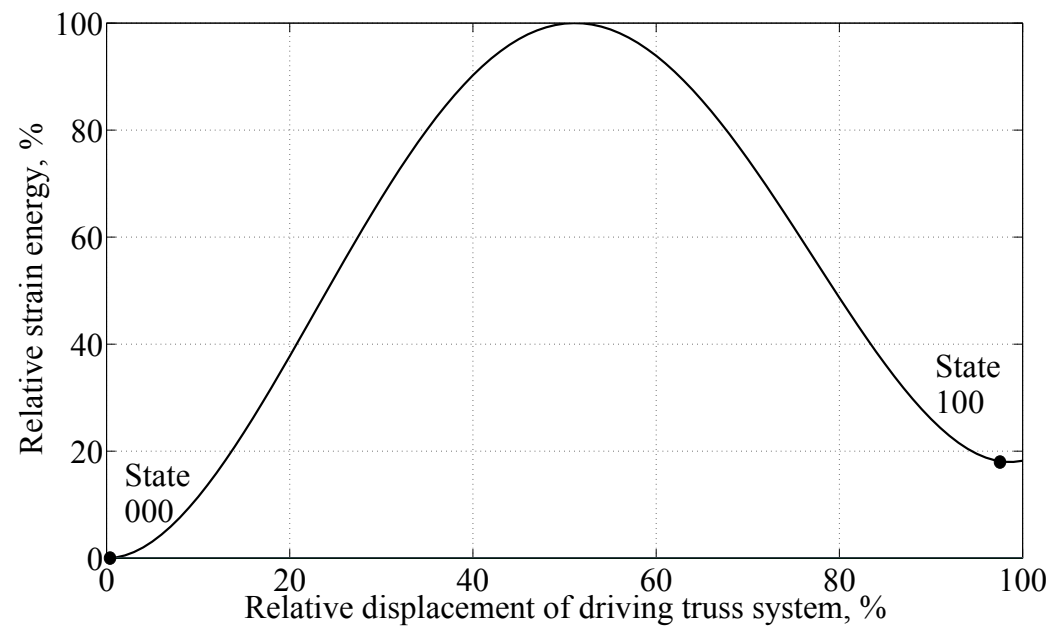

(a) Step A

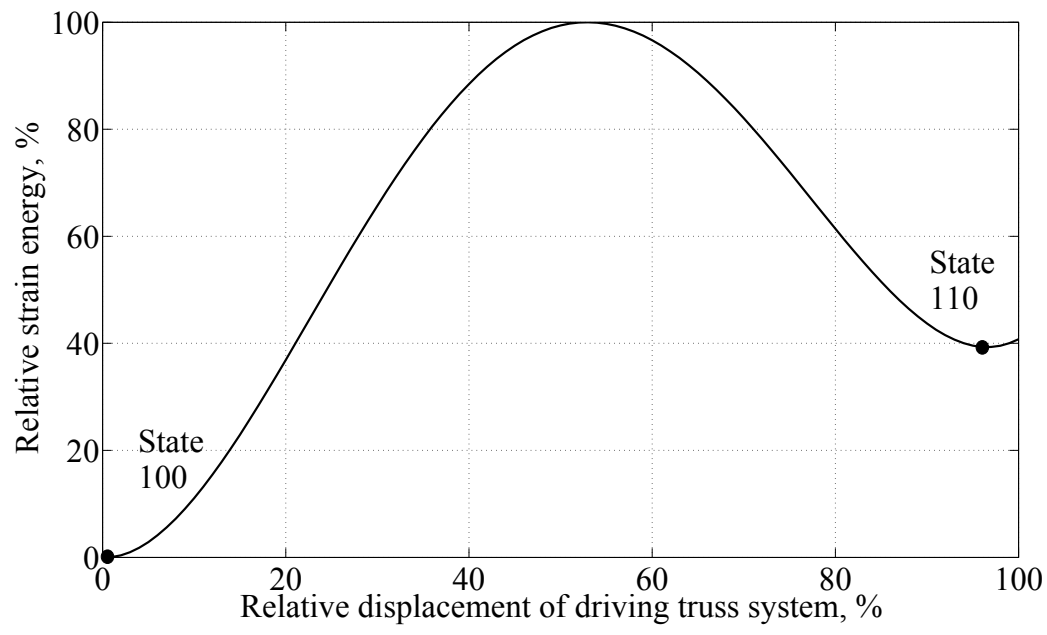

(b) Step B 


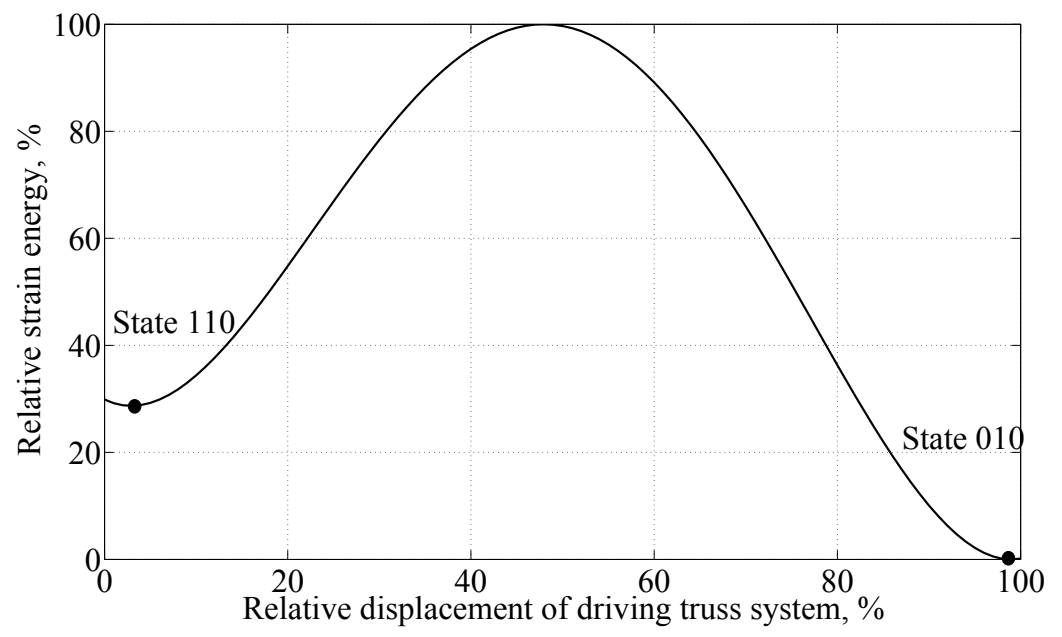

(c) Step C

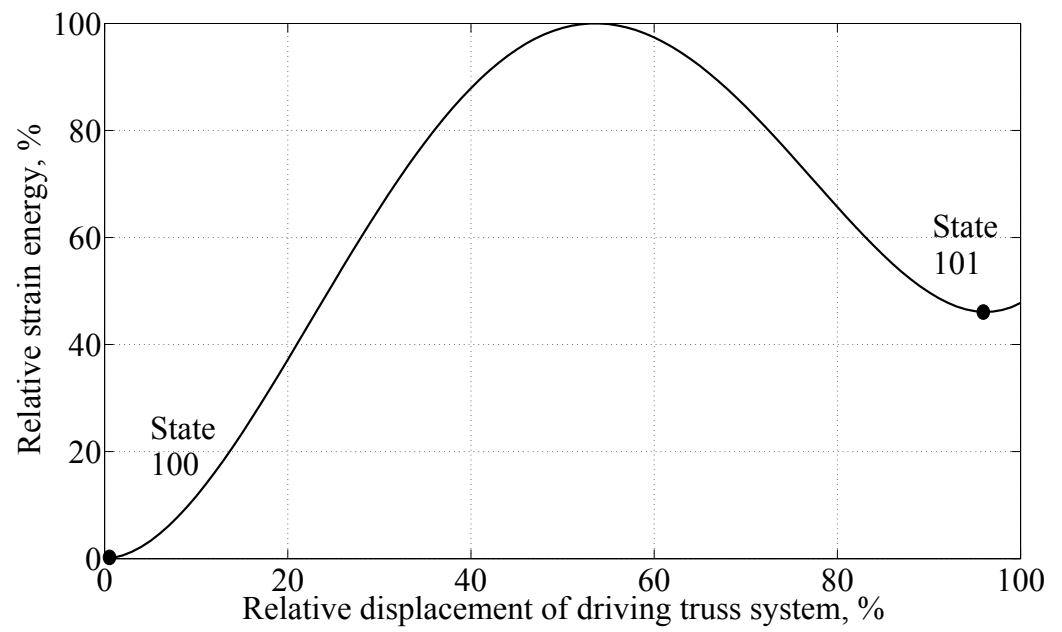

(d) Step D

Figure 6: Relative displacement of trusses in different actuation steps plots for the tripled trusses 


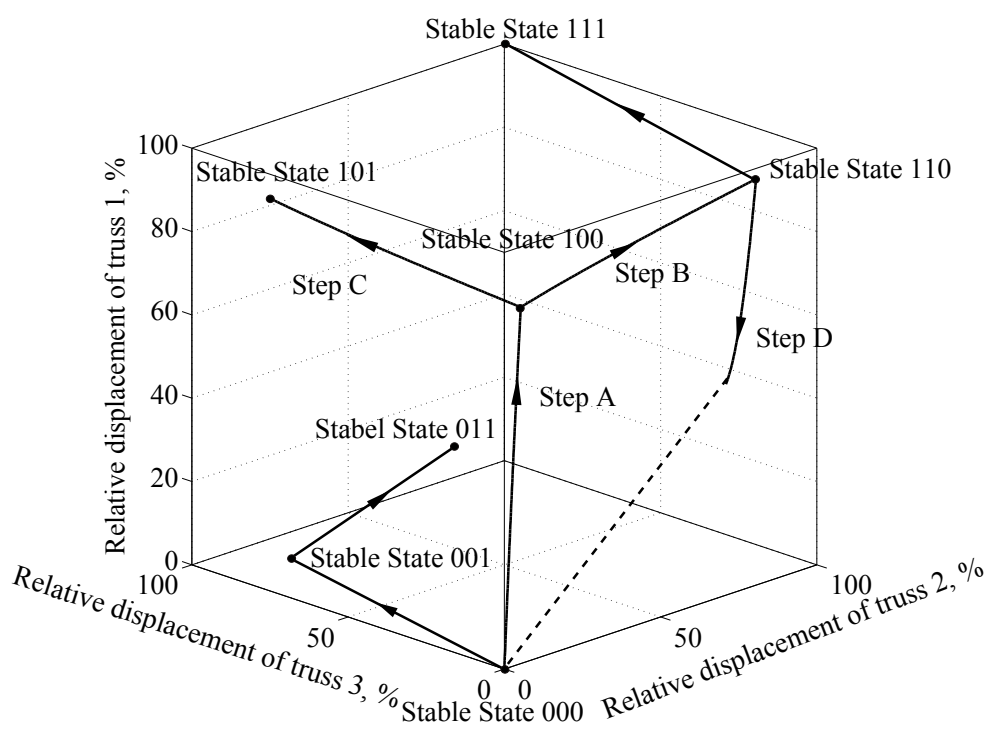

Figure 7: Relative displacement of trusses vs relative strain energy plots for the heptastable truss structure 


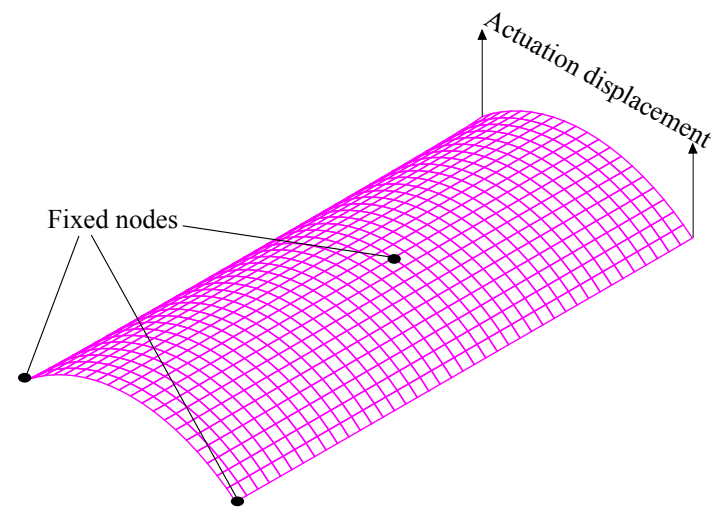

(a) Initial primary stable state (00)

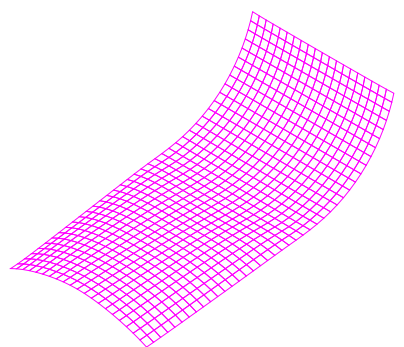

(b) Intermediate state (01)

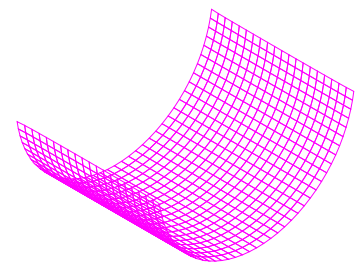

(c) Final primary stable state (11).

Figure 8: Actuation procedure of a coupling bistable composite shells from a primary stable state 




Figure 9: Two different approaches of attaching a biasing strip on a single shell

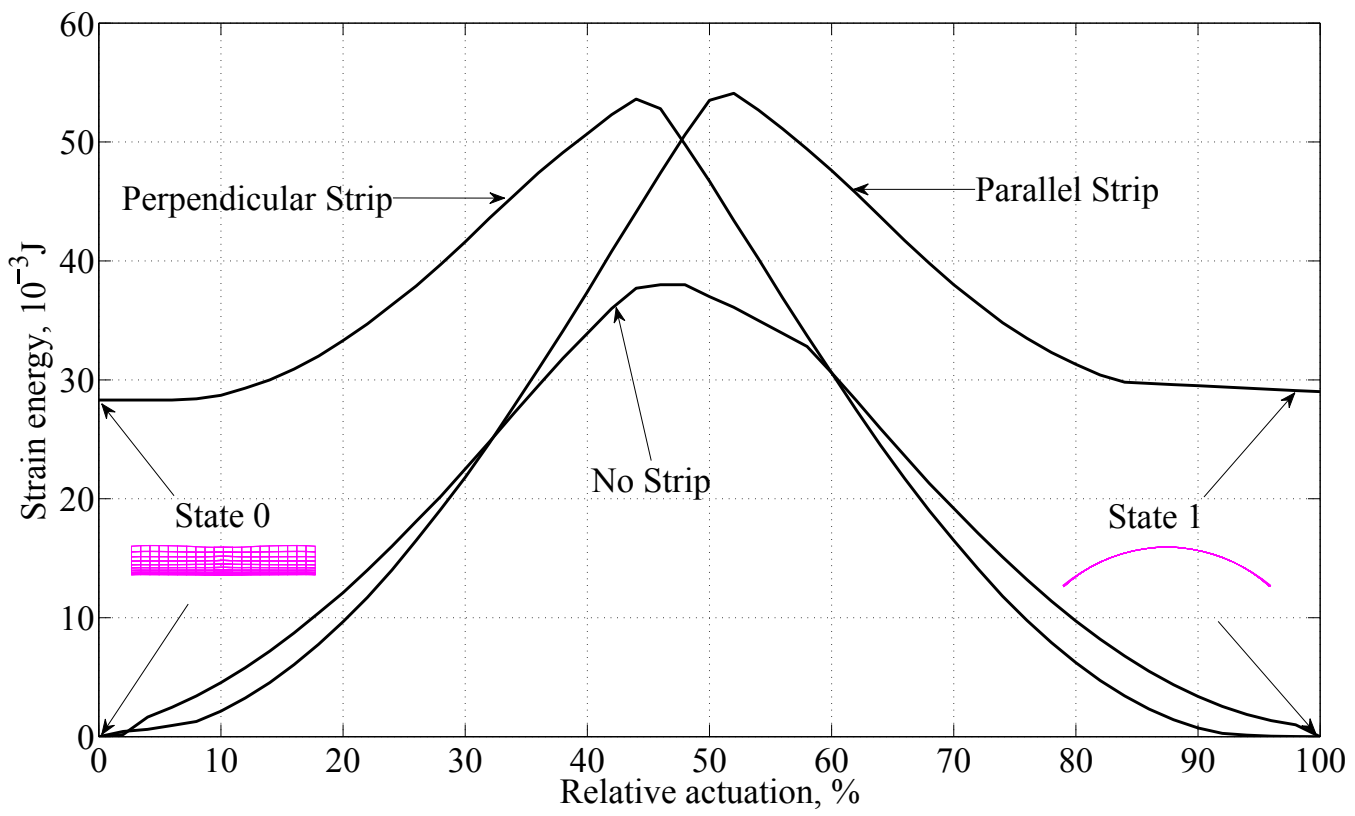

Figure 10: Strain energy vs. relative actuation percentage plots for a symmetric bistable shell and two identical shells with biasing strips attached 


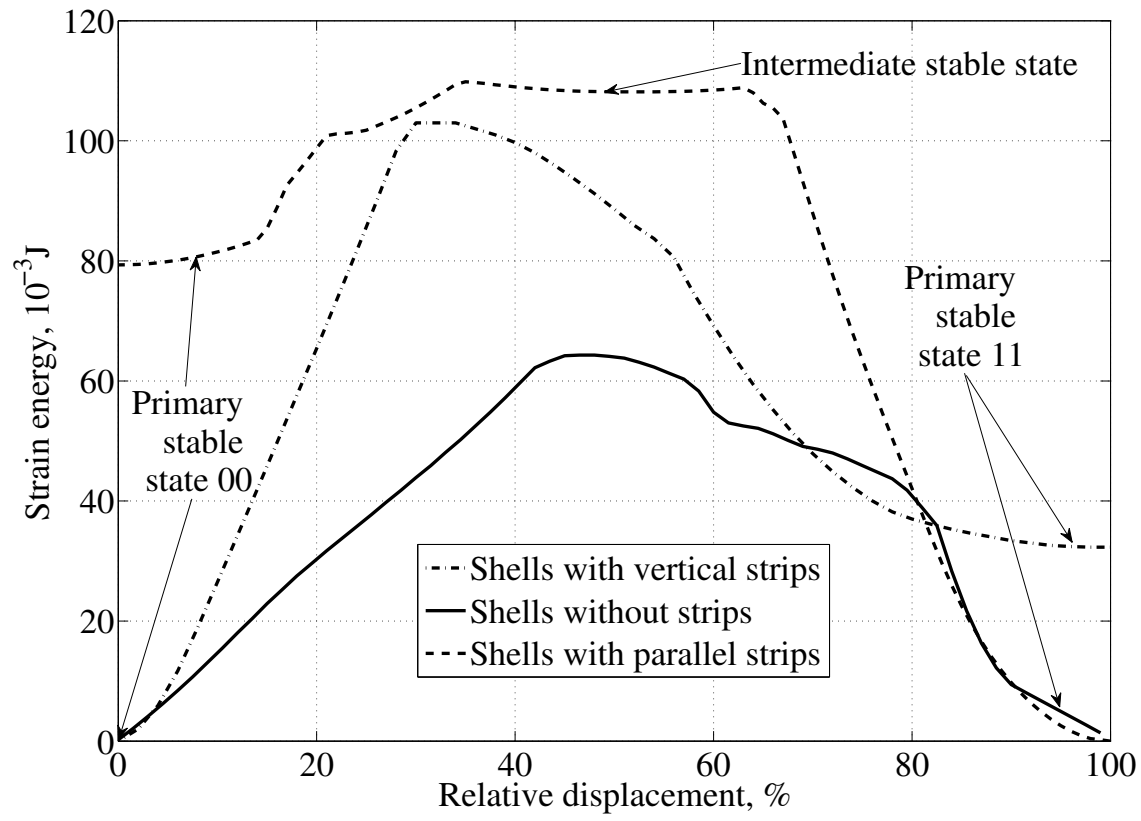

Figure 11: Strain energy vs. relative actuation displacement percentage plots for three different coupled bistable shells 


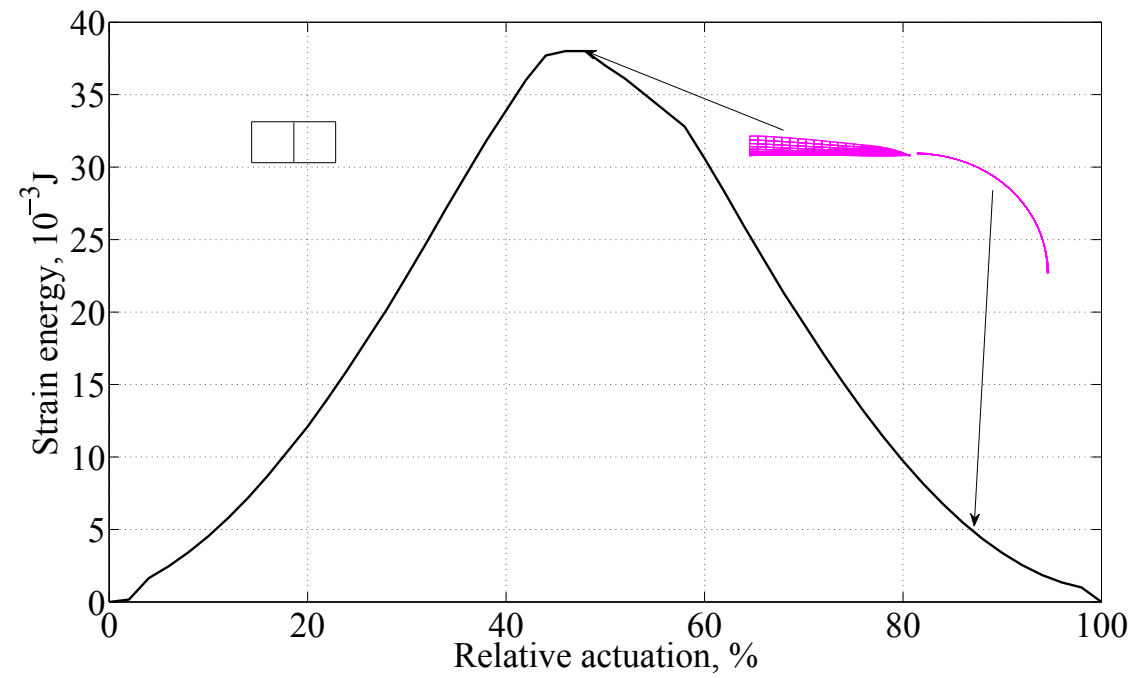

Figure 12: Configurations of two symmetrically bistable shells in an intermediate displacement-controlled state and their corresponding strain energy: this coupled system is bistable



Figure 13: Configurations of two asymmetrically bistable shells in an intermediate displacement-controlled state and their corresponding strain energy: this coupled system is quadstable 


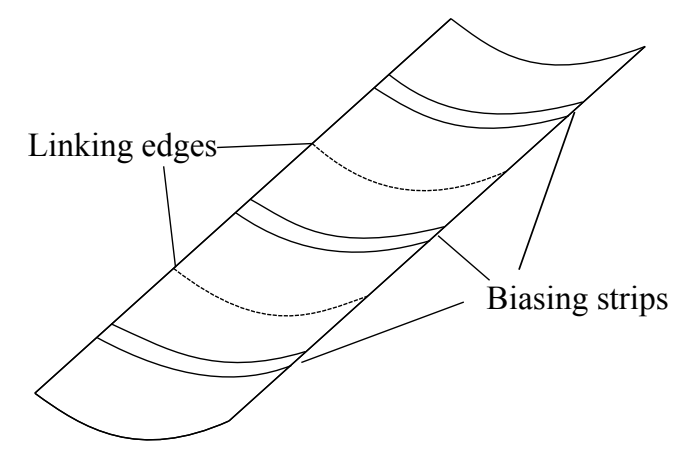

Figure 14: Three connected square shells shown here with biasing strips attached in their parallel orientation 


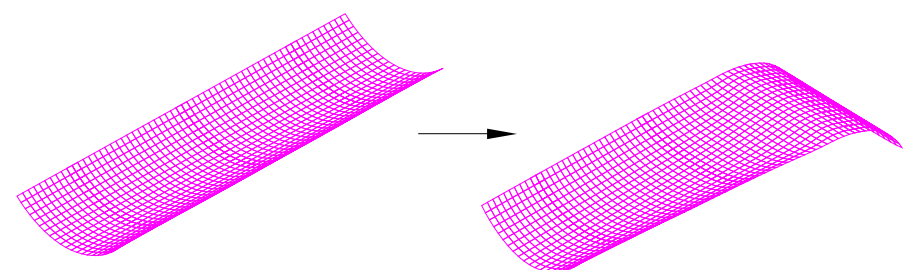

(a) Step A

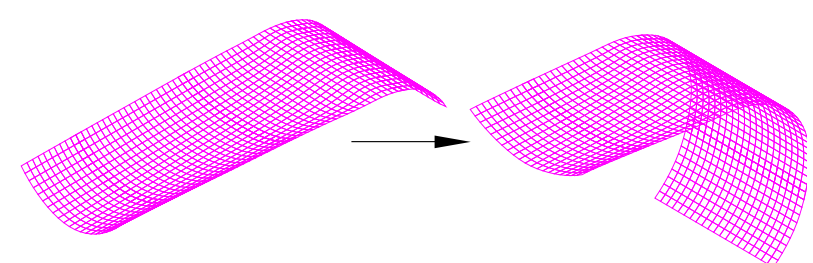

(b) Step B



(c) Step C

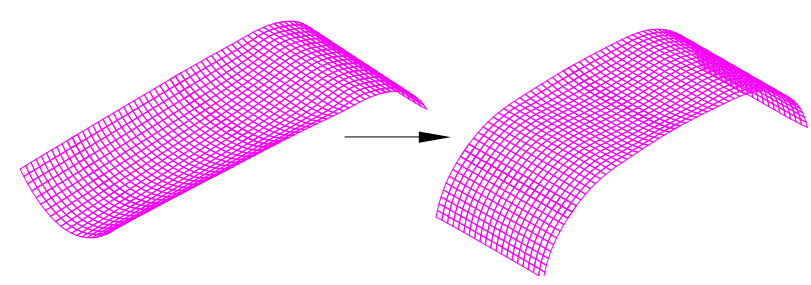

(d) Step D

Figure 15: Relative displacement of trusses in different actuation steps plots for the tripled trusses 


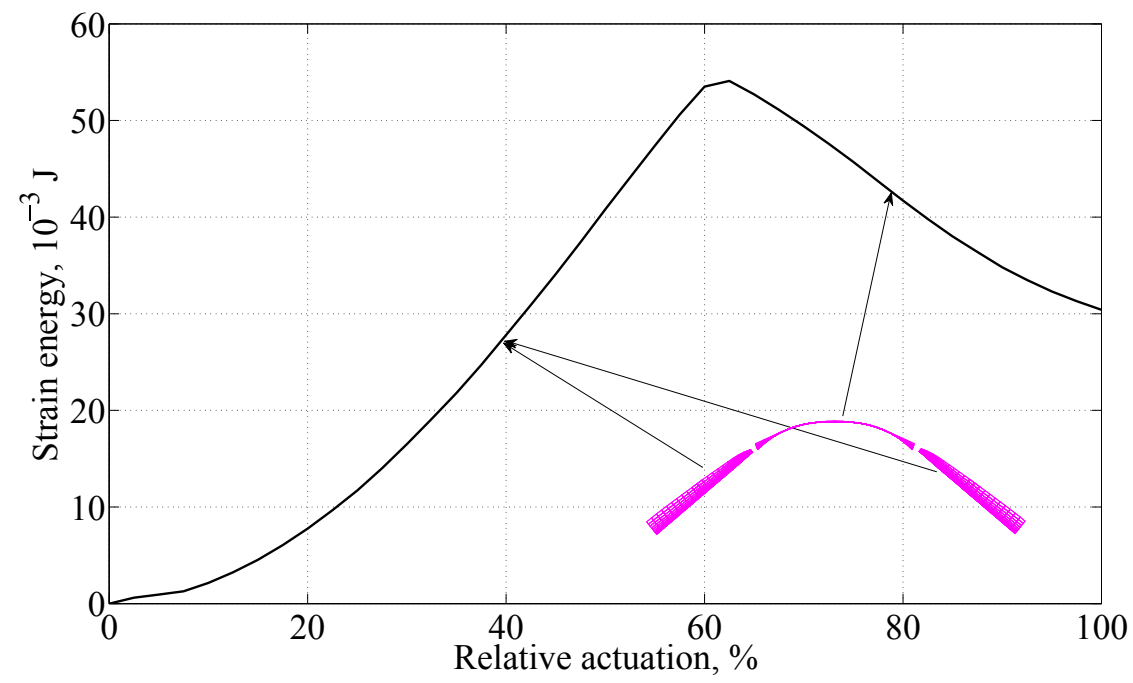

Figure 16: Configurations of three asymmetrically bistable shells in the intermediate displacement-controlled state 010 and their corresponding strain energy: indicates state 010 is stable

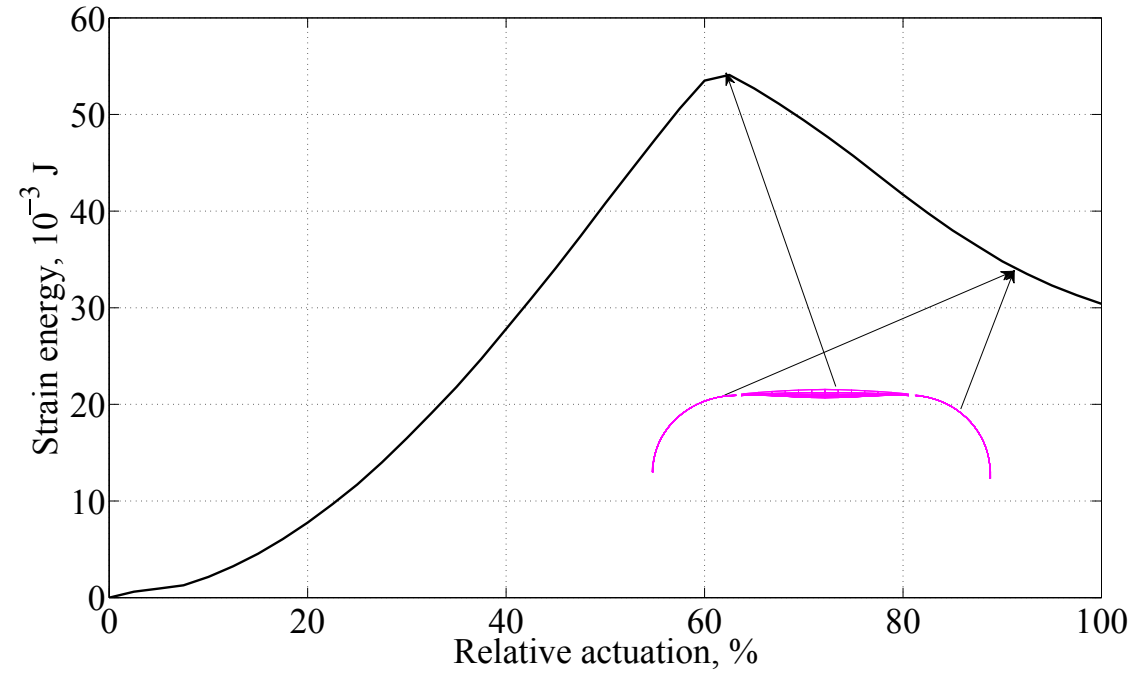

Figure 17: Configurations of three asymmetrically bistable shells in the intermediate displacement-controlled state 101 and their corresponding strain energy: indicates state 101 is unstable 


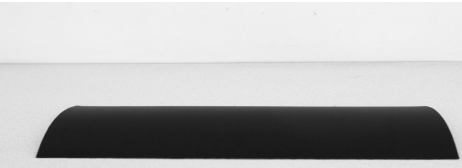

(a) State 000

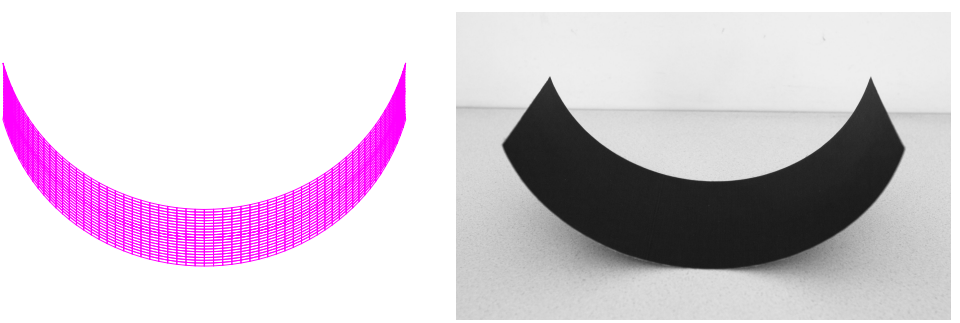

(b) State 111

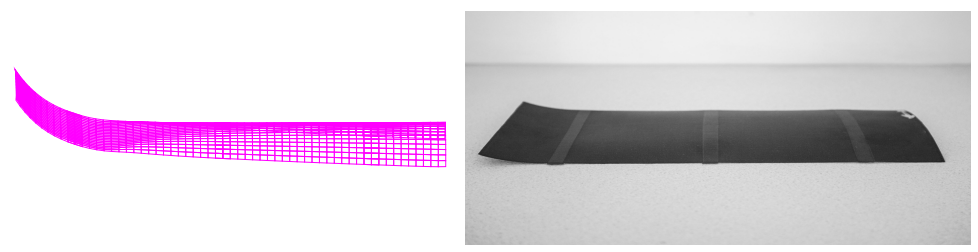

(c) State 001 (also 100)
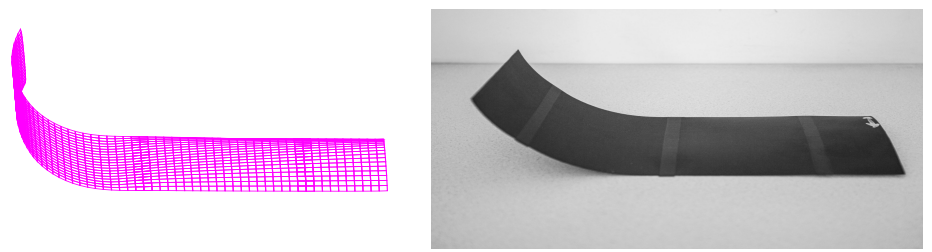

(d) State 011 (also 110)

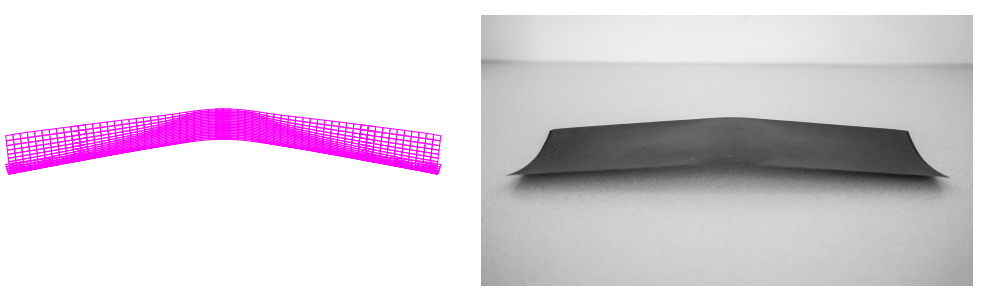

(e) State 010

Figure 18: The maximum seven stable configurations of tripled bistable shells and the FEA results 